\title{
El registro sedimentario de la Cuenca del Bajo Segura (SE España) a través del análisis de correspondencia: implicaciones paleoambientales
}

\section{The sedimentary record of the Bajo Segura basin (SE Spain) through correspondence analysis: paleoenvironmental implications}

\author{
H. Corbí \\ Departamento de Ciencias de la Tierra y del Medio Ambiente. Universidad de Alicante. Campus San Vicente del \\ Raspeig. 03690 San Vicente del Raspeig (Alicante, Spain). Email: hugo.corbi@ua.es; ORCID ID: http://orcid. \\ org/0000-0002-8433-6585
}

\section{RESUMEN}

Se ha analizado el registro sedimentario Messiniense y Plioceno de la Cuenca del Bajo Segura a través del análisis multivariante de correspondencia sin tendencia (DCA). Los resultados obtenidos permiten examinar de forma integral y sintética los ambientes sedimentarios y sintemas representados, atendiendo a la distribución de foraminíferos bentónicos y planctónicos (grupos ecológicos) registrados en seis secciones representativas de la cuenca, tres en el borde norte (Colmenar, Torrellano y Elche), y otras tres en el borde sur (Garruchal, La Pedrera y El Canal). Del análisis estadístico, contrastado con las fuentes bibliográficas disponibles, se puede concluir que: a) el sector sur presenta en comparación con el sector norte, una mayor diversidad de ambientes marinos; y b) los tres sintemas analizados presentan unas características propias, en términos de variedad y representación de los ambientes sedimentarios registrados.

Palabras clave: Crisis de Salinidad del Messiniense; Cordillera Bética; Foraminifera; Análisis multivariante; Paleoambientes.

\begin{abstract}
The Messinian and Pliocene record of the Bajo Segura Basin has been analysed by multivariate detendred correspondence analysis (DCA). The obtained results enables a comprehensive and synthetic examination of the sedimentary record, considering the distribution of benthic and planktic foraminifera (ecological groups) recorded in six representative sections of the basin, three on the northern sector (Colmenar, Torrellano and Elche) and three on the southern part (Garruchal, La Pedrera and El Canal). From the statistical analysis, contrasted with the bibliographical sources available, it can be concluded that: a) the southern sector presents, in comparison with the northern sector, a greater diversity of marine environments; and b) the three analysed synthems have their own characteristics in terms of variety and representation of their registered sedimentary environments.
\end{abstract}

Keywords: Messinian Salinity Crisis; Betic Cordillera; Foraminifera; Multivariate analysis; Paleoenvironments.

Recibido el 22 de junio de 2017; Aceptado el 28 de septiembre de 2017; Publicado online el 21 de noviembre de 2017

Citation / Cómo citar este artículo: Corbí, H. (2017). El registro sedimentario de la Cuenca del Bajo Segura (SE España) a través del análisis de correspondencia: implicaciones paleoambientales. Estudios Geológicos 73(2): e071. https://doi.org/10.3989/ egeol.42908.459.

Copyright: () 2017 CSIC. This is an open-access article distributed under the terms of the Creative Commons Attribution-Non Commercial (by-nc) Spain 3.0 License. 


\section{Introducción}

En estudios micropaleontológicos los métodos estadísticos permiten establecer relaciones y ordenar conjuntos amplios de variables (especies o muestras) que pueden ser interpretadas en función de parámetros o variables paleoambientales (se remite a Murray, 1991, 2006; Shi, 1993; Martin, 2000 para una revisión del tema). De entre los muchos tipos de análisis estadísticos que se pueden implementar, en este trabajo se ha optado por utilizar un análisis de correspondencia (Benzécri, 1992), ya que éste permite visualizar en un mismo gráfico bidimensional el conjunto de datos obtenidos. El análisis de correspondencia sin tendencia (DCA, por sus siglas en inglés Detrended Correspondence Analysis), también denominado rectificado o segmentado, es habitualmente empleado en ecología (Jongman et al., 1995) e incluso en reconstrucciones paleobatimétricas basadas en foraminíferos (e.g. Herkat, 2007; Herkat y Ladjal 2013). Este tipo de análisis se utiliza básicamente para la ordenación de las especies (categorías taxonómicas) y muestras en un mismo gráfico bidimensional. Particularmente, éste se emplea cuando parte de las asociaciones están caracterizadas por frecuencias muy bajas o muchos ceros (Hammer y Harper, 2006). Básicamente el algoritmo DCA genera ejes o factores no correlacionados que maximizan la dispersión de los valores de las especies. La principal ventaja que ofrece esta técnica estadística es que permite visualizar en una misma gráfica las variables y los casos, es decir, las categorías taxonómicas y las muestras. Así pues, el conjunto de datos puede ser analizado de forma integral, estableciendo similitudes y diferencias entre el total de muestras y categorías taxonómicas consideradas.

La pasada década ha sido testigo de un avance considerable en el conocimiento estratigráfico, sedimentológico y micropaleontológico de la Cuenca del Bajo Segura, una de las cuencas neógenas más representativas del Mediterráneo occidental en cuanto a intervalo temporal registrado (desde el Tortoniense al Cuaternario) y variedad de paleoambientes marinos y medios de transición representados (principalmente batial, circalitoral, infralitoral y laguna litoral) (Soria et al., 2008a, 2008b, Caracuel et al., 2011, García-García et al., 2011; Soria et al., 2014; Corbí et al., 2016; Corbí y Soria, 2016; Gamonal et al., 2017; Martínez-Martínez et al., 2017; Corbí et al., 2018, entre otros). Sin embargo, hasta la fecha los análisis estadísticos multivariantes basados en datos cuantitativos de asociaciones fósiles no han sido explorados en esta cuenca. Este tipo de análisis estadístico supone una excepcional herramienta para abordar el análisis paleoecológico y paleoambiental del registro micropaleontológico, tal como ponen de manifiesto las investigaciones realizadas en otros registros neógenos del Mediterráneo (e.g. Gliozzi y Grossi, 2008, Gennari et al., 2013, Walid et al., 2017). Es por ello, que en el presente trabajo se aborda por primera vez el análisis estadístico multivariante (en particular el DCA, mencionado anteriormente) del registro estratigráfico marino y medios de transición, básicamente lagunas litorales de edad Messiniense y Plioceno de la Cuenca del Bajo Segura, tomando como punto de partida las investigaciones previas anteriormente comentadas. La finalidad de este artículo es, por tanto, examinar estadísticamente el registro estratigráfico desde la perspectiva de las unidades aloestratigráficas o sintemas, sistemas de depósito y asociaciones de foraminíferos registrados en dicha cuenca.

\section{Contexto Geológico}

Los materiales analizados pertenecen a la Cuenca del Bajo Segura (Figura 1), una de las cuencas post-orogénicas de la Cordillera Bética (Viseras et al., 2004) que incluye un registro sedimentario desde el Tortoniense hasta la actualidad (Corbí y Soria, 2016). Esta cuenca, ubicada geológicamente en el extremo oriental de la Cordillera Bética, se localiza sobre el contacto entre los dos dominios principales de esta cordillera, las Zonas Externas y las Zonas Internas. El límite norte de la cuenca coincide con la Falla de Crevillente mientras que el sur no está definido dado que esta cuenca se encuentra en continuidad con la de Cartagena. Hacia el oeste los relieves de la Sierra de Orihuela definen el límite con la Cuenca de Fortuna, mientras que, hacia el este, la cuenca se abre hacia el Mediterráneo.

En esta cuenca es posible distinguir los sectores, norte y sur, claramente definidos desde el punto de vista tectónico y paleogeográfico (Figuras 1 y 2). Ambos sectores están separados por el valle fluvial del río Segura, cuya génesis esta relacionada con la actividad reciente de la Falla del Bajo Segura. En el sector norte el basamento de la cuenca lo forman los 
(A)

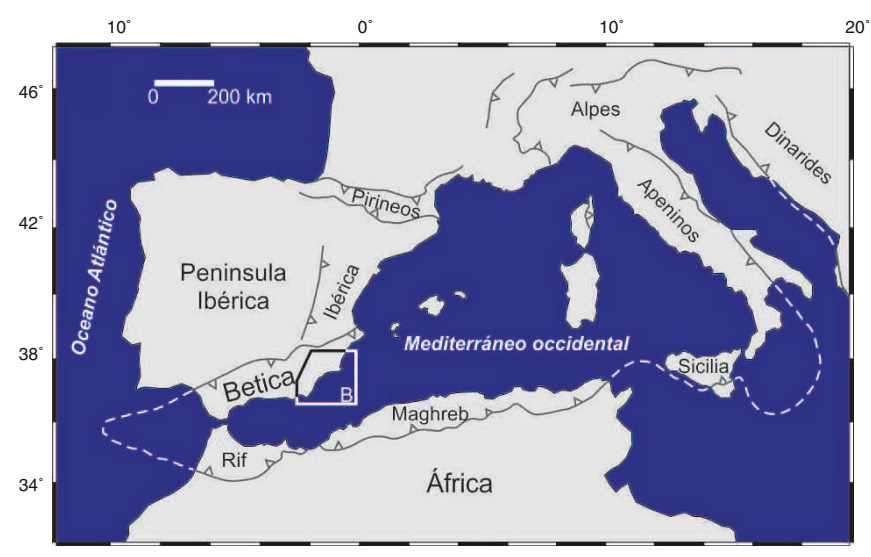

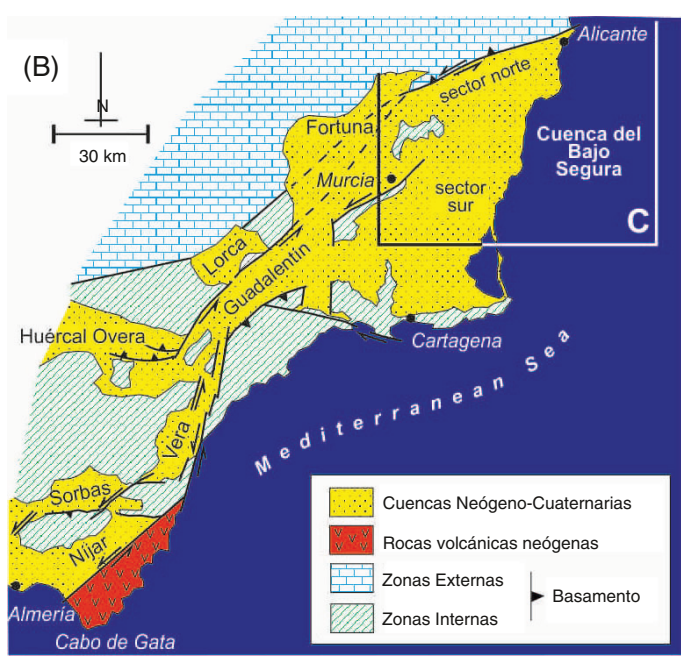

Fig. 1.-Localización geológica de la Cuenca del Bajo Segura (modificado de Corbí et al., 2016).

materiales de las Zonas Externas, mientras que en el sector sur está constituido por materiales de las Zonas Internas. La estructura de estos sectores presenta notables diferencias, de modo que en el sector norte los materiales definen un dispositivo monoclinal con frecuentes discordancias progresivas asociadas al movimiento de la Falla de Crevillente y al levantamiento de los relieves de las Zonas Externas. En cambio, en el sector sur, los materiales se encuentran afectados por pliegues, entre los que destaca, por sus proporciones, el Anticlinal de Torremendo. Desde el punto de vista paleogeográfico estos dos sectores también muestran ciertas diferencias. En el sector norte las unidades aloestratigráficas presentan, el líneas generales, asociaciones de facies típicas de medios marinos someros y continentales; en cambio, en el sector sur, estas unidades se caracterizan por presentar sistemas de depósito marinos de mayor profundidad y espesor (Corbí, 2010; Corbí y Soria, 2016).

Trabajos recientes dividen el registro marino de esta cuenca en 5 unidades aloestratigráficas o sintemas: Tortoniense I, Tortoniense II, TortonienseMessiniense I, Messiniense II y Plioceno (Soria et al., 2008a, 2008b, Corbí y Soria, 2016). Estas unidades quedan, por tanto, separadas por discontinuidades representadas por superficies erosivas que corresponden con cambios paleogeográficos y tectonosedimentarios. Las figuras 3 y 4 ilustran el mapa geológico sintético, así como la organización estratigráfica para los sectores norte y sur de esta cuenca. En todo el registro sedimentario se ha documentado la completa sucesión de marcadores bioestratigráficos de foraminíferos planctónicos, desde la biozona MMi9 (Tortoniense inferior) al MP13 (Plioceno inferior) (Corbí y Soria, 2016). Se refiere a Soria et al., 2008a, 2008b, Corbí et al. (2016), Corbí y Soria (2016) y Corbí (2010) para una descripción exhaustiva de las principales características estratigráficas, sedimentológicas y micropaleontológicas de las unidades representadas en esta cuenca. Es de destacar que, cada una de las unidades definidas contienen diferentes conjuntos de litofacies y biofacies $\mathrm{y}$, por lo tanto, comprenden más de una unidad litoestratigráfica y bioestratigráfica. De acuerdo con estos autores, el análisis de los diferentes conjuntos de facies que integran estas unidades ha permitido diferenciar sistemas de depósito (sensu Fisher y McGowan, 1967) que cubren un amplio espectro de ambientes sedimentarios, desde batiales a lagunares (Figuras 3 y 4 ).

\section{Materiales y técnicas estadísticas}

En este trabajo se ha realizado un análisis de correspondencia sin tendencia (DCA) en el conjunto de datos de distribución de foraminíferos representados en 139 muestras de 6 secciones estratigráficas. Para llevar a cabo este procedimiento estadístico se empleo el programa informático PAST C (Hammer et al., 2001). Una primera caracterización tafonómica de la asociación registrada pone de manifiesto 


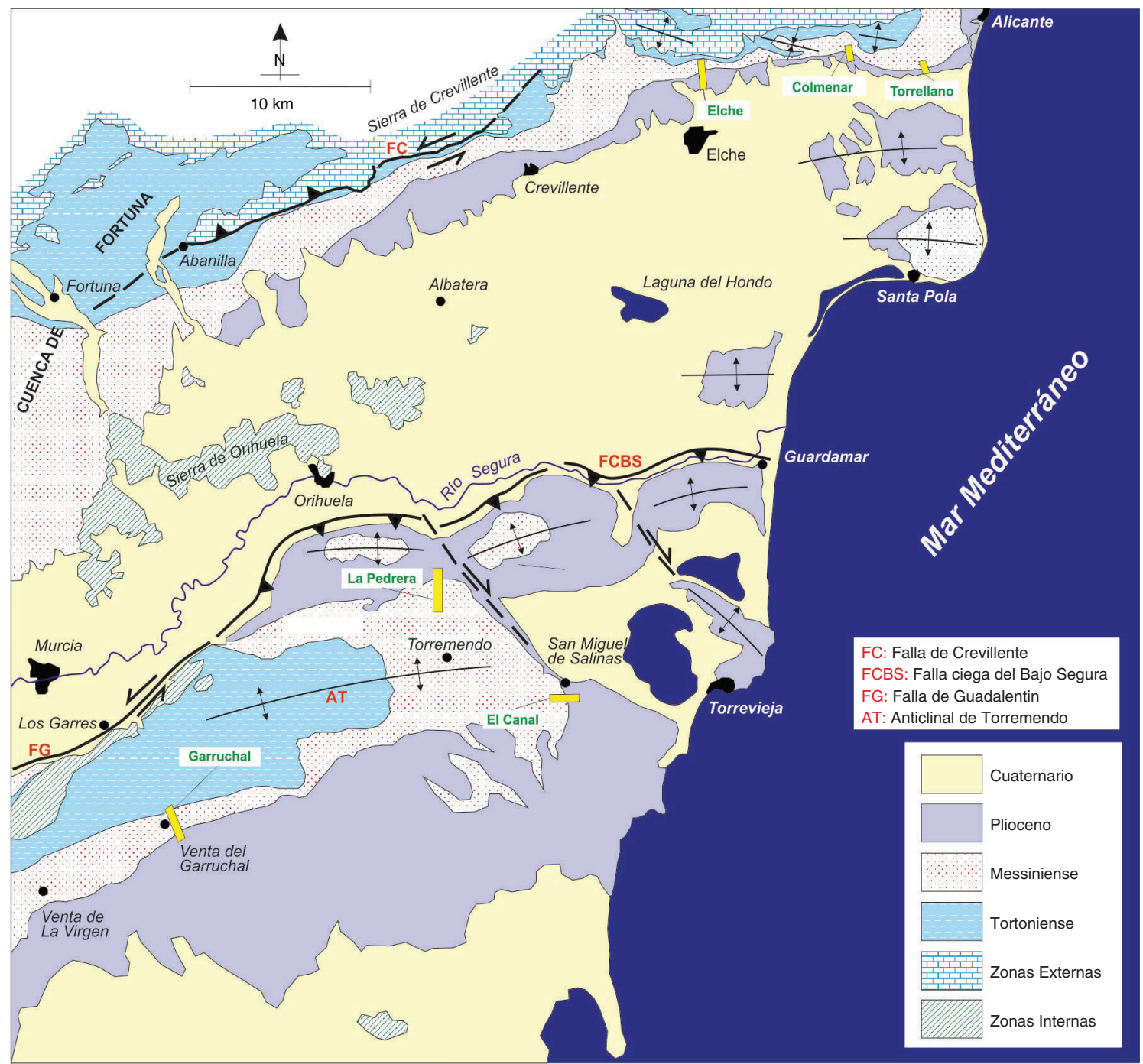

Fig. 2.-Mapa geológico sintético de la cuenca del Bajo Segura con ubicación de las secciones estratigráficas estudiadas (modificado de Corbí y Soria, 2016).

que las conchas de la mayor parte de los foraminíferos analizadas presentan un buen estado de conservación (Corbí, 2010). Por tanto, en líneas generales las asociaciones pueden considerarse como acumuladas (sensu Fernández-López, 2000). No obstante, en algunos casos puntuales se han detectado foraminíferos que presentan signos de alteración como fracturas, perforaciones, perdida de transparencia y formación de concreciones lo cual podría ser indicativo de resedimentación y/o reelaboración.

Se eligió el análisis de correspondencia sin tendencia, ya que es el habitualmente utilizado cuando se tiene presencia de varias especies en las asociaciones de foraminíferos caracterizadas por bajas frecuencias. Además, permite representar en un mismo gráfico muestras y grupos ecológicos de foraminíferos bentónicos y planctónicos, lo cual facilita un análisis integral de toda la cuenca. Para ello, se han considerado los datos cuantitativos de distribución de asociaciones de foraminíferos bentónicos y planctónicos representados en los sistemas de depósito marinos y de medios de transición, principalmente lagunares, de edad Messiniense y Plioceno de las secciones representativas del sector norte (Elche, Torrellano y Colmenar) y del sector sur (Garruchal, La Pedrera y El Canal) de la Cuenca del Bajo Segura (véase Figuras 5 y 6 para localización de las muestras analizadas). Las muestras fueron previamente 
(A)

(B)
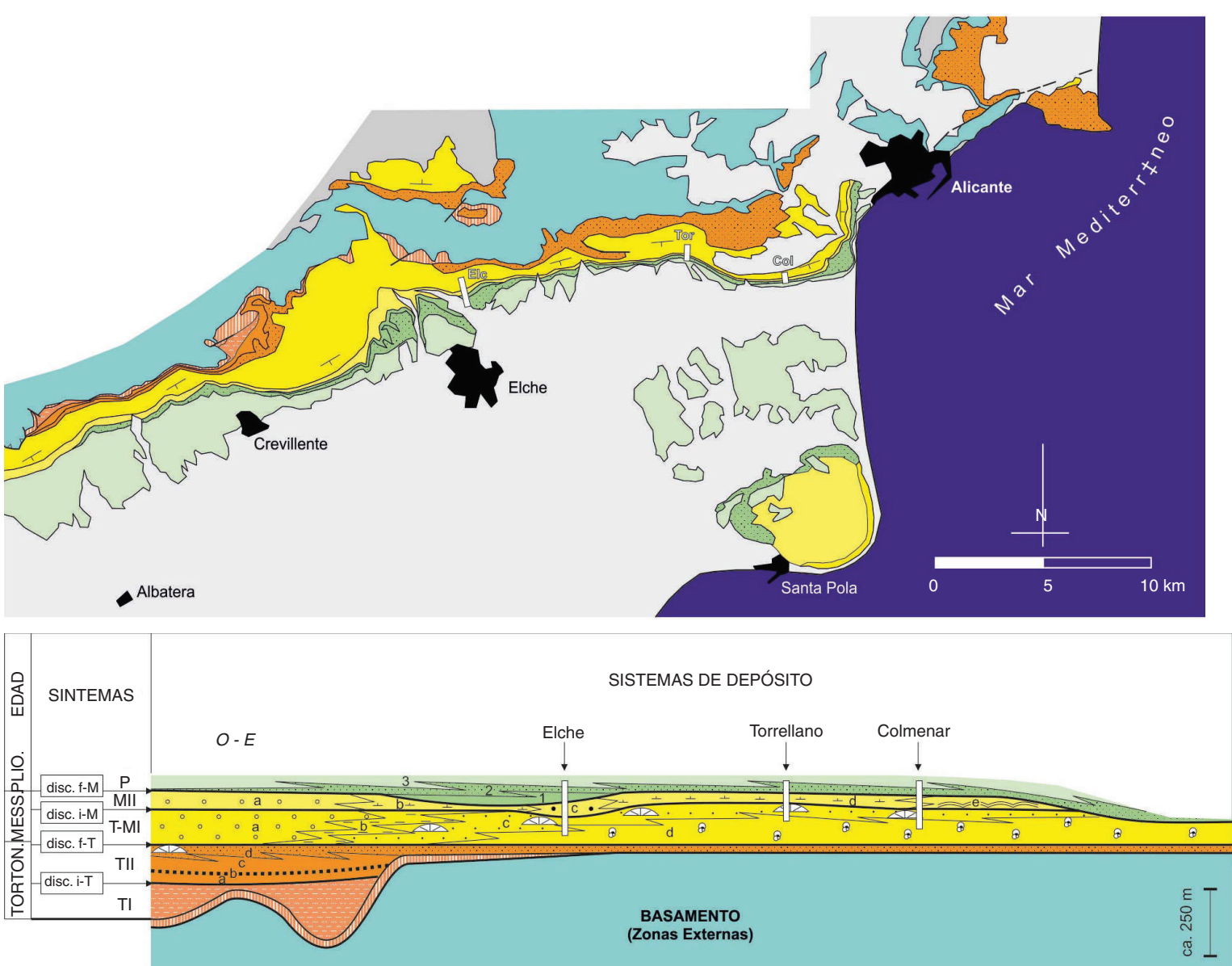

Fig. 3.-Mapa geológico sintético (A) y organización estratigráfica (B) del sector norte de la cuenca, con ubicación de las secciones estudiadas (modificado de Corbí, 2010).

levigadas de acuerdo al procedimiento convencional, tras lo cual se analizó semicuantitativamente la fracción contenida entre los tamices de 125 y 500 $\mu \mathrm{m}$, contabilizando y determinando a nivel de especie (siempre que fuera posible) un total de 300 ejemplares de foraminíferos por muestra (Corbí, 2010).

Con el propósito de analizar de forma integral, completa y sintética el registro marino y lagunar litoral representado, se han establecido grupos ecológicos de foraminíferos bentónicos (GEFB) y planctónicos (GEFP), tomando como punto de partida los datos cuantitativos de distribuciones de foraminíferos (porcentajes de representación) presentados en Corbí (2010). Los grupos ecológicos establecidos corresponden a conjuntos de especies de foraminíferos característicos de un determinado ambiente marino. Por tanto, en la matriz de datos generada para el análisis DCA, las asociaciones establecidas (grupos ecológicos) representan los "casos", y las muestras, las "variables". Esta agrupación ha permitido visualizar e integrar los datos a distintas escalas, como pueden ser los sectores de la cuenca, sintemas, secciones estratigráficas y sistemas de depósito (Figura 7).

Los grupos ecológicos de foraminíferos bentónicos (GEFB) considerados, expresados en la Tabla 1, están relacionados tanto con el ambiente marino como con el microhábitat (modo de vida). En esta tabla se muestran las especies representadas en la Cuenca del Bajo Segura agrupadas según el morfogrupo y microhábitat al que pertenecen. Respecto al microhábitat conviene señalar que los dos grupos establecidos, epifaunal-infaunal somero e infaunal profundo, son los propuestos por Jorissen et al. (1995). No obstante, y con el propósito de sintetizar los resultados, los grupos ecológicos de foraminíferos 
(A)

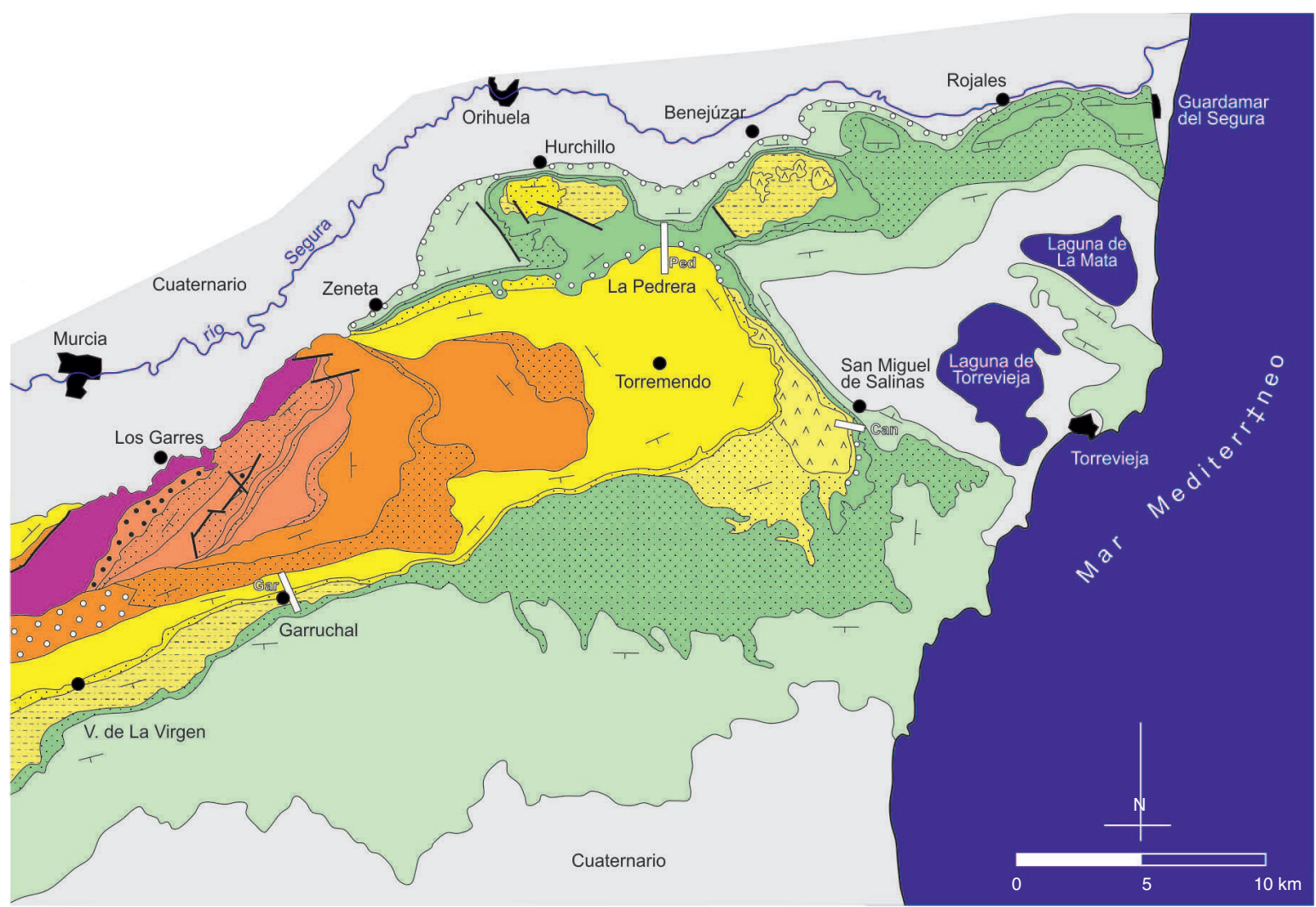

(B)

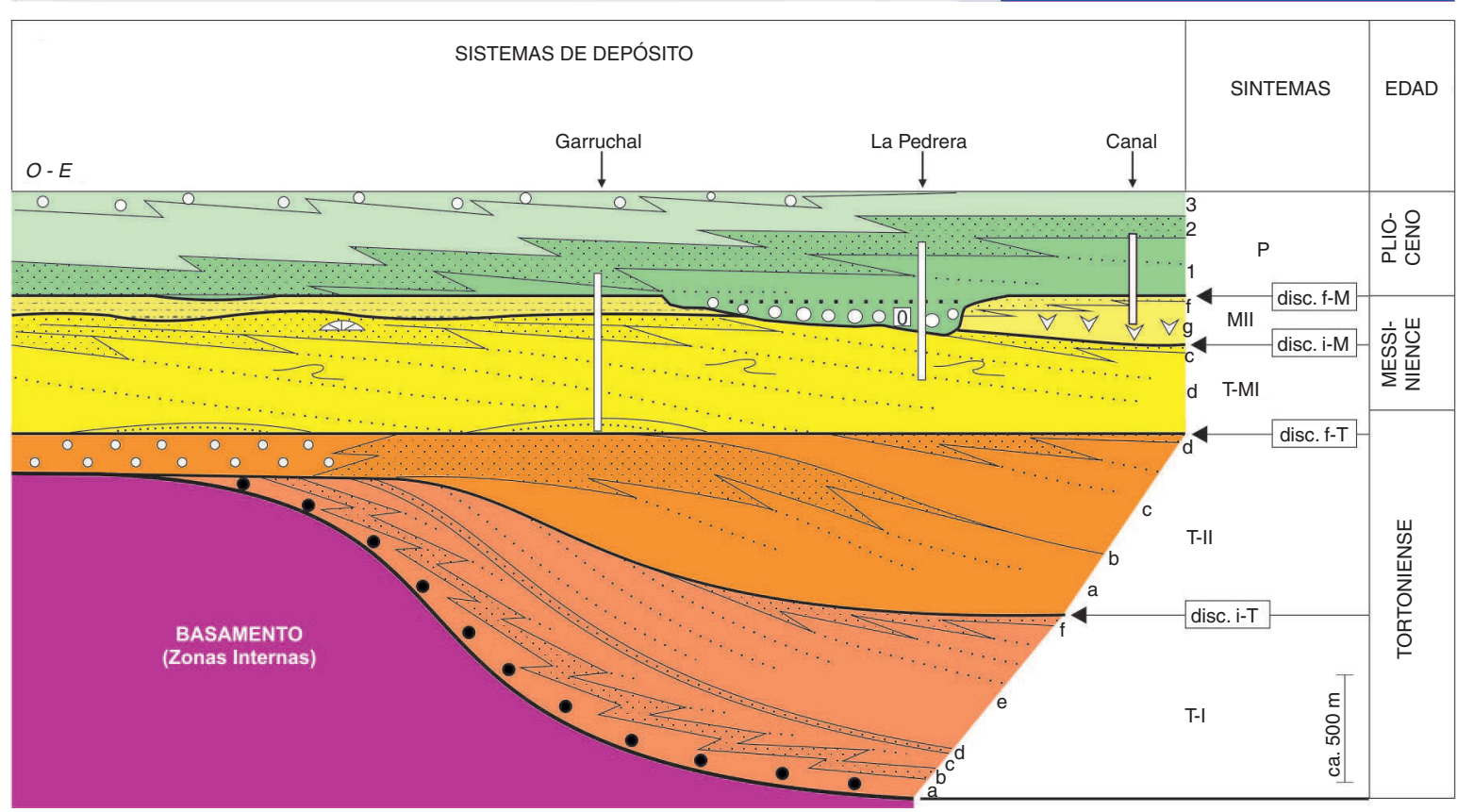

Fig. 4.-Mapa geológico sintético (A) y arquitectura estratigráfica (B) del sector sur de la cuenca. Se incluye la localización de las secciones analizadas (modificado de Corbí, 2010).

bentónicos (tabla 1) han sido agrupados y sintetizados en representativos de ambientes de tipo lagunar (L), infralitoral (I), circalitoral (C) y batial (B). El ambiente sedimentario característico de cada especie ha sido obtenido de la información autoecológica bibliográfica disponible, fundamentalmente de 


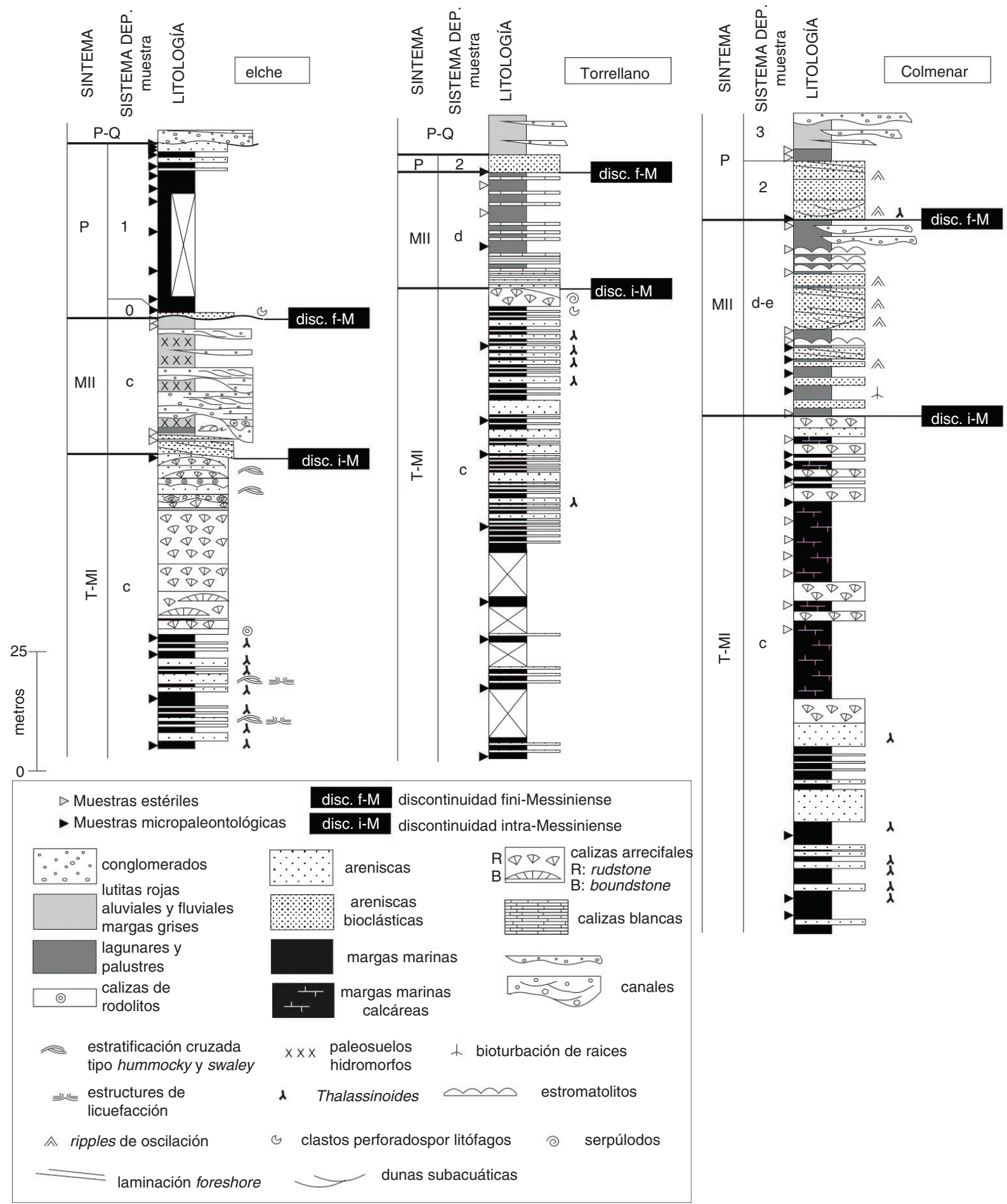

Fig. 5.- Secciones estratigráficas representativas del sector norte de la cuenca con ubicación de las muestras estudiadas (modificado de Corbí, 2010).

Murray (1991 y 2006). Ahora bien, cabe señalar que, para ciertos autores, algunas de estas especies poseen un rango posible de distribución más amplio del que se ha considerado en este trabajo, en el que sólo se ha tenido en cuenta su ambiente de más habitual. Así pues, las distribuciones de cada uno de estos grupos 


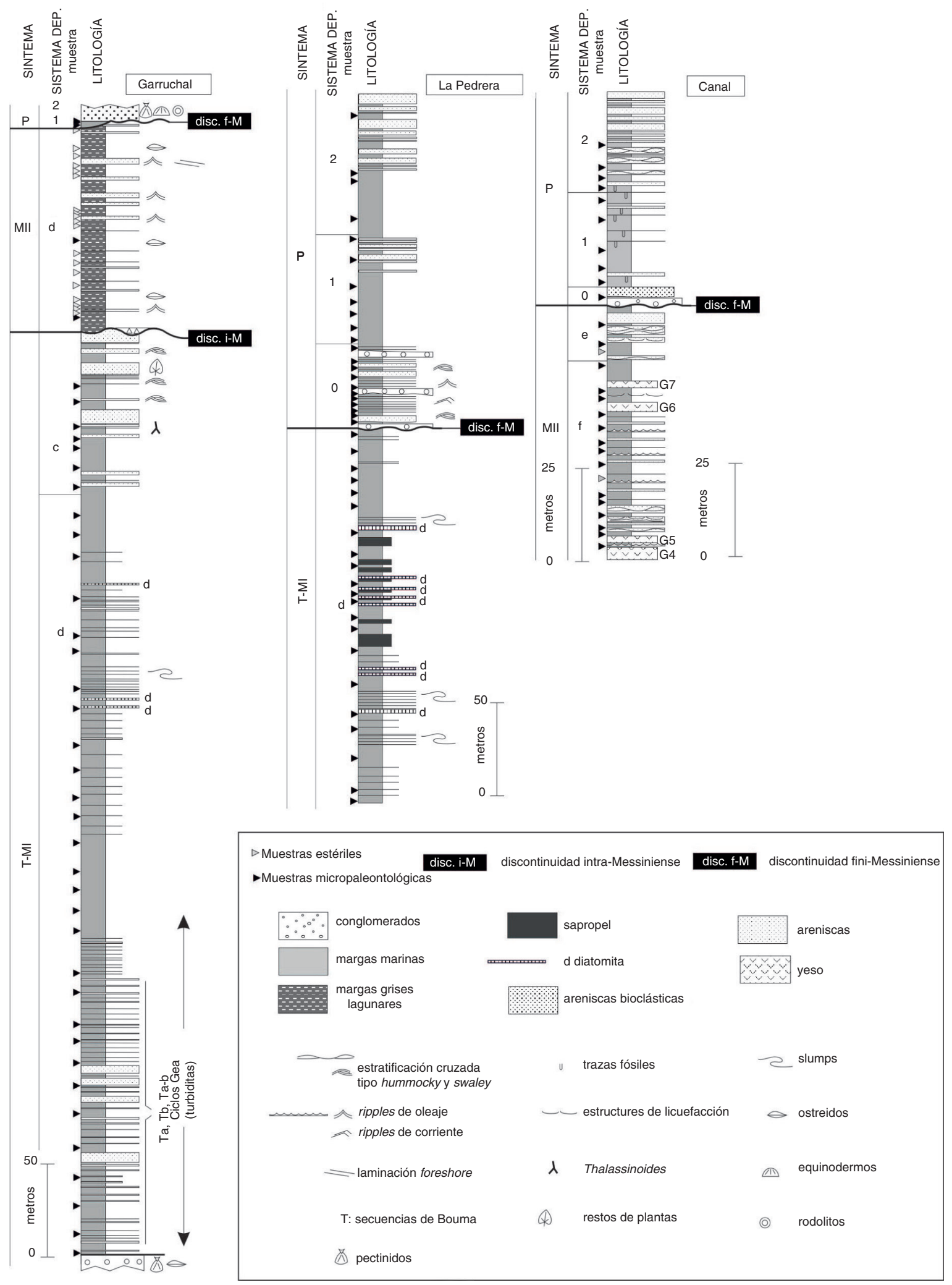

Fig. 6.-Secciones estratigráficas representativas del sector sur de la cuenca con ubicación de las muestras estudiadas (modificado de Corbí, 2010). 


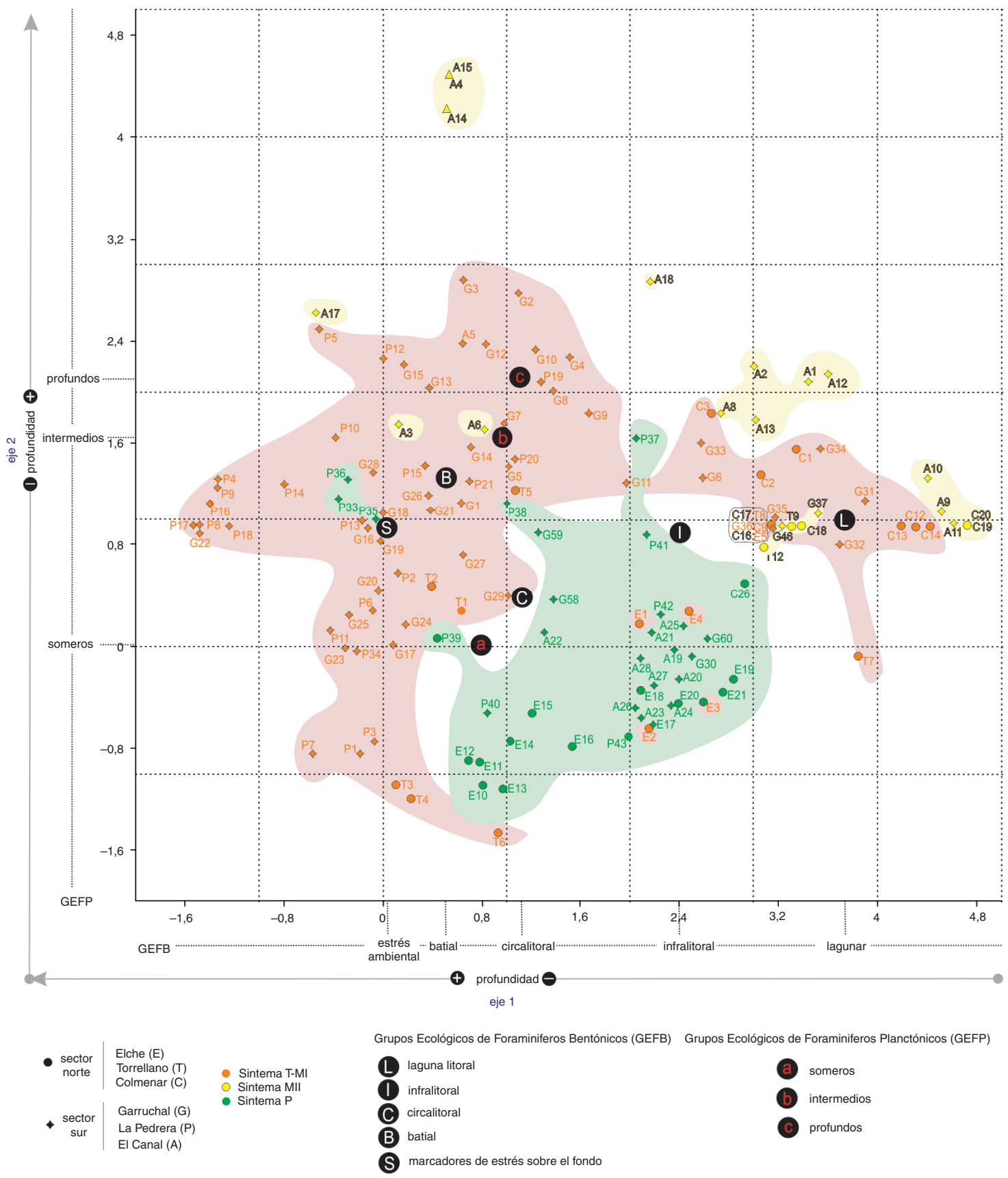

Fig. 7.-Análisis de correspondencia sin tendencia (DCA) basado en grupos ecológicos de foraminíferos para las secciones representativas del registro Messiniense y Plioceno de la Cuenca del Bajo Segura. Obsérvese que, con el propósito de visualizar más sintéticamente las muestras, se ha coloreado las regiones que incluían muestras de la misma unidad.

representan, en términos generales, el ambiente predominante en cada una de las muestras analizadas.

Por otra parte, el contenido en oxígeno es uno de los factores fundamentales que controlan la distribución de los foraminíferos bentónicos, además de encontrarse relacionado directamente con la salinidad, temperatura y circulación de la masa de agua (Van der Zwaan et al., 1999). Numerosos estudios ponen de manifiesto una relación directa entre el grado de oxigenación de las aguas y el modo de vida de los foraminíferos bentónicos, de tal forma que, generalmente, en condiciones de baja 
Tabla 1.-Grupos ecológicos de foraminíferos bentónicos considerados en el análisis estadístico, y modo de vida de cada uno de los taxones representados.

\begin{tabular}{|c|c|c|c|}
\hline $\begin{array}{l}\text { Grupos ecológicos } \\
\text { (GEFB) }\end{array}$ & \multicolumn{2}{|l|}{ Microhábitat } & Foraminíferos bentónicos \\
\hline Laguna litoral (L) & \multicolumn{2}{|l|}{ A } & $\begin{array}{l}\text { Ammonia tepida, Elphidium granosum, Elphidium } \\
\text { williamsoni y Haynesina germanica }\end{array}$ \\
\hline \multirow[t]{5}{*}{ Infralitoral (I) } & \multicolumn{2}{|c|}{ B1: epifaunal-infaunal somero } & $\begin{array}{l}\text { Ammonia inflata, Ammonia beccari, Aubignyna mariei y } \\
\text { Amphistegina lessonii }\end{array}$ \\
\hline & \multirow[t]{4}{*}{ B2: epifítícos } & $\begin{array}{l}\text { B2a: epifítico, adherido } \\
\text { permanentemente }\end{array}$ & $\begin{array}{l}\text { Cibicides lobatula, Cibicides refulgens y Planorbulina } \\
\text { mediterranensis }\end{array}$ \\
\hline & & $\begin{array}{l}\text { B2b: epifítico, adherido } \\
\text { temporalmente }\end{array}$ & $\begin{array}{l}\text { Asterigerinata mamila, Biasterigerina planorbis, } \\
\text { Cymbaloporetta squamosa y Rosalina spp. }\end{array}$ \\
\hline & & B2c: epifitico, libre (Elphidium) & $\begin{array}{l}\text { Elphidium aculeatum, Elphidium advenum, Elphidium } \\
\text { complanatum, Elphidium crispum y Elphidium } \\
\text { macellum }\end{array}$ \\
\hline & & $\begin{array}{l}\text { B2d: epifítico, libre (miliólidos y } \\
\text { Textularia) }\end{array}$ & $\begin{array}{l}\text { Anomalina pompilloides, Globulina spinosa, } \\
\text { Quinqueloculina oblonga y Textularia spp. }\end{array}$ \\
\hline \multirow[t]{2}{*}{ Circalitoral (C) } & \multicolumn{2}{|c|}{ C1: epifaunal (óxico) } & $\begin{array}{l}\text { Anomalinoides flinti, Cancris auriculus, Cibicides } \\
\text { dutemplei, Cibicides pseudoungerianus, Cibicides } \\
\text { ungerianus, Gyroidina spp., Hanzawaia boueana, } \\
\text { Hoeglundulina elegans, Melonis spp., Neoeponides } \\
\text { schreibersii, Nonion commune, Oridorsalis } \\
\text { umbonatus, Sigmoilina tenuis, Sigmoilopsis } \\
\text { schlumbergeri, Siphonina planoconvexa y } \\
\text { Valvulineria complanata }\end{array}$ \\
\hline & \multicolumn{2}{|c|}{ C2: infaunal (dysóxico) } & $\begin{array}{l}\text { Allomorphina trigona, Amphicoryna spp., Bigenerina } \\
\text { nodosaria, Bolivina spp., Bulimina aculeata, } \\
\text { Bulimina echinata, Bulimina elongata, Cassidulina } \\
\text { spp., Chistomella czizeki, Clavulina angularis, } \\
\text { Dentalina spp., Dorothia gibbosa, Eggerella } \\
\text { bradyi, Favulina hexagona, Fursenkoina } \\
\text { scherbesiana, Glandulina laevigata, Globobulimina } \\
\text { spp., Globocassidulina spp., Marginulina spp., } \\
\text { Martinotiella communis, Nonionella turgida, } \\
\text { Planularia crassis, Plectofrondicularia spp., } \\
\text { Pseudonodosaria aequalis, Reusella spinulosa, } \\
\text { Siphotextularia affinis, Spiroplectinella spp., Trifarina } \\
\text { spp., Uvigerina bononiensis y Uvigerina peregrina }\end{array}$ \\
\hline \multirow[t]{2}{*}{ Batial (B) } & \multicolumn{2}{|c|}{ D1: epifaunal (óxico) } & $\begin{array}{l}\text { Cibicides bradyi, Ehrenbergina alicantina, Lenticulina } \\
\text { spp., Planulina ariminensis y Saracenaria latinfrons }\end{array}$ \\
\hline & \multicolumn{2}{|c|}{ D2: infaunal (dysóxico) } & $\begin{array}{l}\text { Bulimina costata, Karreriella bradyi, Pulleina spp., } \\
\text { Sphaeroidina bulloides, Uvigerina cylindrica, } \\
\text { Uvigerina proboscidea, Uvigerina rutila y Uvigerina } \\
\text { striatissima }\end{array}$ \\
\hline
\end{tabular}

oxigenación predominan, en mayor o menor grado, los taxones de carácter infaunal profundo, denominados también como marcadores de estrés ambiental (Hinsbergen et al., 2005). Así pues, de acuerdo con este autor, el conjunto de los foraminíferos bentónicos puede subdividirse en taxones de aguas marinas abiertas "normales" y marcadores o indicativos de estrés ambiental (stress markers). Este último grupo, de gran importancia en la reconstrucción de las concentraciones de oxígeno, está constituido por las especies siguientes: Bolivina spp. (excepto B. plicatella Cushman, 1930 y B. pseudoplicata Heron- Allen \& Earland, 1930), Bulimina spp. (excluidas las especies costuladas); Cancris spp., Chistomella spp., Fursenkoina spp., Globobulimina spp., Rectuvigerina spp., Stainforthia spp., Uvigerina spp. (se excluye $U$. semiornata) y Valvulineria spp. En definitiva, en el presente trabajo, con propósito de conocer el grado de estrés ambiental sobre el fondo de cada unidad, se ha considerado como variable el 
porcentaje de distribución de los foraminíferos bentónicos marcadores de estrés ambiental $\mathrm{S}$, en sentido de Hinsbergen et al. (2005) que representa el porcentaje de marcadores de estrés ambiental sobre el fondo con respecto al total de la asociación de foraminíferos bentónicos.

Los grupos ecológicos de foraminíferos planctónicos (GEFP) considerados están relacionados directamente con la profundidad. Según Bè (1977) se pueden establecer tres grupos principales de foraminíferos planctónicos de acuerdo con su distribución batimétrica (aguas someras, intermedias y profundas). Atendiendo a la clasificación propuesta por este autor, en este trabajo se han considerado los siguientes grupos: a) especies de aguas someras ( $<50$ metros de profundidad), grupo que incluye todas las especies de los géneros Globigerinoides y Turborotalita; b) especies de aguas intermedias (entre 50 y 100 metros de profundidad). Formado por las especies Globigerina bulloides d'Orbigny, 1826, Globigerinella spp., Orbulina universa d'Orbigny, 1839 y Globigerinita naparimaensis Brönnimann, 1951; y c) especies de aguas profundas ( $>100$ metros de profundidad) representado por todas las especies de Globorotalia, Sphaeroidinellopsis spp., y Neogloboquadrina acostaensis (Blow, 1959).

\section{Resultados y discusión}

El análisis multivariante realizado (DCA) ha permitido distribuir las muestras (casos) y los grupos ecológicos (variables: bentónicos, GEFB, planctónicos, GEFP) en un mismo gráfico bidimensional, expresado en la figura 7. Estos resultados estadísticos permiten establecer similitudes y diferencias entre el conjunto de datos estudiados, atendiendo principalmente a las siguientes cuestiones: a) distribución y dominancia de grupos ecológicos en los sintemas representados en la cuenca: TortonienseMessiniense, T-MI, en figura 7 en rojo, Messiniense II, MII, en amarillo y Plioceno, P, en verde; b) comparación sectores norte y sur de la cuenca (secciones Elche, Colmenar y Torrellano en el sector norte; secciones de Garruchal, La Pedrera y Canal en sector sur); y c) rasgos particulares (distribución de grupos ecológicos) de las secciones analizadas y sistemas de depósito representados.
La figura 7 muestra la ordenación bidimensional del total de datos analizados, de modo que se puede realizar una serie de afirmaciones estadísticas. A continuación se exponen y analizan las principales cuestiones representadas en el grafico, discutiendo los resultados en el contexto de los trabajos publicados hasta la fecha que abordan el estudio estratigráfico y micropaleontológico de la Cuenca del Bajo Segura.

\section{El gráfico DCA como síntesis del registro marino de la cuenca}

La distribución de muestras y grupos ecológicos en la gráfica generada permite formular que los ejes del mismo (Figura 7) están directamente relacionados con los tipos ambientes marinos, y por tanto con la profundidad a la que viven los distintos grupos ecológicos establecidos. De esta forma, cada eje (eje horizontal: bentónicos, eje vertical: planctónicos) representa distintos términos de profundidad (distal/profundo - proximal/somero) en la cuenca. De acuerdo con ello, en el eje X pueden ser ubicados (prolongando hasta el eje cada grupo ecológico de bentónicos) los grupos ecológicos de foraminíferos bentónicos (de distal a proximal; batial -B-, circalitoral -C-, infralitoral -I- y lagunar -L-), de modo que conforme aumentan los valores de $\mathrm{X}$, disminuye la profundidad (mayor grado de proximalidad). Además, el grupo indicador de estrés ambiental (S) queda ubicado en el extremo izquierdo del eje $\mathrm{X}$, de tal forma que valores próximos o inferiores a 0 indican condiciones de estrés ambiental sobre el fondo.

Por otra parte, en el eje Y quedan situados, de acuerdo con su profundidad, los grupos ecológicos de foraminíferos planctónicos (someros -a-, intermedios -b-, y profundos -c-), de tal forma que conforme aumentan los valores del eje Y aumenta la profundidad. Además, del gráfico también se desprende que, en líneas generales, las muestras más próximas a la variable S (grupo ecológico de foraminíferos bentónicos representativos de estrés ambiental) presentarán un mayor grado de estrés ambiental. En síntesis, esta interpretación de ejes, permite visualizar en un mismo gráfico la totalidad secciones, muestras y sectores, de acuerdo al grado de proximalidad (aguas más someras) o distalidad (aguas profundas) de los ambientes marinos representados en la cuenca. 


\section{Consideraciones estratigráficas y paleoambientales}

La interpretación del análisis de correspondencia permite establecer una serie de consideraciones que pueden ser interpretadas en términos paleoambientales en relación a los dos sectores paleogeográficos definidos en la cuenca (norte y sur). El gráfico DCA (Figura 7) índica como las muestras pertenecientes a las secciones representativas del borde sur de la cuenca (Garruchal, La Pedrera y Cana) se encuentran presentes en una mayor superficie del gráfico. En cambio, las muestras representativas de las secciones del borde norte (Elche, Torrellano y Colmenar) se encuentran ubicadas principalmente en la parte inferior derecha del gráfico, quedando representadas fundamentalmente por grupos ecológicos de foraminíferos bentónicos de ambientes circalitoral, infralitoral y lagunar, junto con foraminíferos planctónicos típicos de aguas someras e intermedias. Conforme a estas inferencias estadísticas es posible concluir que el sector sur presenta, en comparación con el sector norte, un mayor grado de variabilidad de ambientes sedimentarios marinos y medios de transición (laguna litoral), así como una mayor cantidad de muestras que registran ambientes de aguas profundas (batiales).

Esta interpretación estadística del gráfico es coherente con la organización estratigráfica y la interpretación sedimentológica y paleoambiental propuesta para los dos sectores de la cuenca (Soria et al., 2008a, 2008b; Corbí, 2010; Caracuel et al., 2014; Corbí y Soria, 2016; Corbí et al., 2016). De acuerdo con estos autores, los paneles de organización estratigráfica para los dos sectores (expresados en las figuras 5 y 6 ) ponen de manifiesto, en particular para el sintema Tortoniense-Messiniense I un mayor grado de diversidad de ambientes marinos, observado especialmente en un mayor desarrollo de los ambientes marinos profundos (batiales) en el sector sur de la cuenca. Este argumento queda reflejado especialmente en la sección de Garruchal, que registra en la base de este sintema, sedimentación turbidítica, ambiente sedimentario no registrado en ningún punto de las unidades Messiniense II y Plioceno (Soria et al., 2008a).

Por otra parte, y también en este mismo Sintema (Tortoniense-Messiniense I), la distribución de las muestras en el gráfico refleja una mayor dominancia de estrés ambiental (muestras muy próximas a la variable $\mathrm{S}$, estrés ambiental), en comparación, por ejemplo con las muestras pertenecientes al Plioceno. Este estrés ambiental se pone de manifiesto especialmente en las muestras próximas a los niveles diatomíticos (Figura 6; análisis de facies en Soria et al., 2008a), donde existen niveles prácticamente dominados por foraminíferos bentónicos marcadores de estrés ambiental (baja oxigenación) como Bulimina o Globobulimina.

El gráfico muestra también que las muestras pertenecientes al sintema MII (muestras en amarillo en Figura 7, sección Canal principalmente) quedan próximas a los grupos ecológicos de foraminíferos bentónicos típicos de ambiente lagunar (L) e infralitoral (I). Además, las muestras representadas en esta sección parecen contener una mayor dispersión en la gráfica; de hecho, se observan muestras ubicadas en los extremos del gráfico. Estos dos rasgos estadísticos son congruentes con el hecho de que en la sección Canal, el Sintema Messiniense II, constituido principalmente por margas inter-estratificadas con yesos (Unidad sin-evaporítica) registra fauna enana de foraminíferos planctónicos, junto con especies de foraminíferos bentónicos de carácter eurihalino y estenohalino (Corbí et al., 2016). Según estos autores, esta asociación característica índica condiciones anómalas en la columna de agua relacionadas con cambios de salinidad importantes, en un contexto de cuenca marina con estratificación de la columna de agua y precipitación evaporítica episódica. En consecuencia, aunque existe un conjunto importante de muestras muy próximas a la variable "ambiente lagunar/infralitoral" típica de este contexto, también la situación anómala de esta unidad impide la coherente interpretación de la misma dentro del gráfico estadístico generado, incluyendo muchas muestras en puntos alejados de la gráfica.

Finalmente, el sintema Plioceno (muestras en verde en figura 7) incluye muestras muy próximas a las variables de grupos ecológicos de bentónicos dominados por faunas típicas de ambientes circalitorales e infralitorales, así como próximas también a los grupos ecológicos de planctónicos de aguas someras intermedias. Es de destacar, que en líneas generales no se registran en ningún punto de la cuenca ambientes de aguas profundas (batiales), como también ponen de manifiesto Corbí et al., 2016 y Corbí y Soria (2016). No obstante, el gráfico también indica, como un 
conjunto de muestras de este sintema de la sección del Garruchal (ubicadas a la izquierda del gráfico) representan las muestras de carácter más profundo de todo el Plioceno. Además, este conjunto de muestras se encuentran ubicadas también próximas a la variable $\mathrm{S}$ (estrés ambiental), y quedan representadas en la parte inferior de la unidad Plioceno de la sección La Pedrera. De acuerdo con Corbí et al. (2016), esté estrés ambiental podría interpretarse en términos de condiciones de baja oxigenación sobre el fondo (circulación limitada de oxígeno), probablemente relacionadas con la propia configuración de la cuenca al inicio del Plioceno: bahías y paleovalles reinundados tras la Crisis de Salinidad Messiniense que representan ambientes relativamente restringidos.

\section{Conclusiones}

Este estudio pretende demostrar como el análisis multivariante, en particular el análisis de correspondencia sin tendencia, representa un instrumento estadístico eficaz para el análisis integral de toda una cuenca sedimentaria basado en asociaciones de foraminíferos. Este trabajo supone un primer punto de partida para la exploración futura de otros procedimientos estadísticos de detalle que contemplen otros aspectos relevantes en la interpretación de paleoambientes según las asociaciones de foraminíferos registradas, como, por ejemplo, las variaciones en el estado de conservación (gradientes tafonómicos) o la variedad intraespecífica entre distintas especies.

Este análisis estadístico, implementado aquí por primera vez para el registro marino de la Cuenca del Bajo Segura, permite extraer las siguientes conclusiones, siendo todas ellas contrastadas con la información bibliográfica de carácter estratigráfico, sedimentológico y micropaleontológico disponible:

a) Los ejes del gráfico DCA generado, y que explican la mayor parte de la varianza, pueden ser interpretados en términos de grado de proximalidad/ aguas someras - distalidad/aguas profundas de los ambientes sedimentarios representados en la cuenca.

b) El sector sur de la cuenca registra una mayor variedad de ambientes sedimentarios, así como, en líneas generales ambientes de aguas más profundas de carácter batial.

c) El sintema Tortoniense - Messiniense I (preevaporítico) registra mayor diversidad de ambientes sedimentarios, desde batiales a lagunares. En el sintema Messiniense II predominan los ambientes de carácter lagunar - infralitoral, y muestras anómalas, rasgo característico de la unidad syn-evaporítica. El sintema Plioceno presenta menos dispersión, dominando los ambientes de carácter infralitoral y circalitoral.

\section{AGRADECIMIENTOS}

Este trabajo se ha realizado en el marco del grupo de investigación Cambios Paleoambientales de la Universidad de Alicante (vigrob-167) y los proyectos GRE14-05 (Universidad de Alicante) y CGL2016-79878-R (Ministerio de Economía y Competitividad de España). El autor agradece a Jesús M. Soria (Universidad de Alicante), su guía y apoyo en las diferentes fases de este trabajo. El autor agradece los comentarios realizados por Juan Usera Mata y Matías Reolid, que han permitido mejorar este trabajo.

\section{Referencias}

Bé, A.W.H. (1977). An ecological, zoogeographic and taxonomic review of recent planktonic foraminifera. In: Oceanic Micropaleontology (Ramsay, A.T.S., Ed.), Academic Press, London, 1-100.

Benzécri, J.P. (1992). Correspondence analysis handbook. Marcel Dekker, 688 pp.

Caracuel, J.E.; Corbí, H.; Giannetti, A.; Monaco, P.; Soria, J.M.; Tent-Manclús, J.E. \& Yébenes, A. (2011). Paleoenvironmental changes during the Late Miocene (Messinian)-Pliocene transition: sedimentological and ichnological evidence. Palaios, 26: 754-766. https://doi.org/10.2110/palo.2011.p11-016r

Corbí, H. (2010). Los foraminíferos de la cuenca neógena del Bajo Segura (sureste de España): bioestratigrafía y cambios paleoambientales en relación con la Crisis de salinidad del Mediterráneo. Tesis Doctoral, Universidad de Alicante, $252 \mathrm{pp}$.

Corbí, H.; Fierro, I.; Aberasturi, A. \& Sánchez Ferris, E.J. (2018). Potential use of a significant scientific geosite: The Messinian coral reef of Santa Pola (SE Spain). Geoheritage, In press. https://doi. org/10.1007/s12371-017-0268-6

Corbí H.; Soria, J.M.; Lancis C.; Giannetti A.; Tent-Manclús J.E. \& Dinarès-Turell, J. (2016). Sedimentological and paleoenvironmental scenario before, during, and after the Messinian Salinity Crisis: The San Miguel de Salinas composite section (western Mediterranean). Marine Geology, 379: 246-266. https:// doi.org/10.1016/j.margeo.2016.05.017 
Corbí, H. \& Soria, J.M. (2016). Late Miocene-early Pliocene planktonic foraminifer event-stratigraphy of the Bajo Segura basin: A complete record of the western Mediterranean. Marine and Petroleum Geology, 77: 1010-1027. https://doi.org/10.1016/j. marpetgeo.2016.08.004

Fernández-López, S. (2000). Temas de tafonomía. Departamento de Paleontología, Facultad de Ciencias Geológicas, Universidad Complutense de Madrid.

Fisher, W.L. \& McGowan, J.H. (1967). Depositional systems in the Wilcox of Texas and their relationship to occurrence of oil and gas. Transactions of the Gulf Coast Association of Geological Societies, 17: 105-125.

Gamonal, A.; Mansino, S.; Ruiz-Sánchez, F.J.; Crespo, V.D.; Corbí, H. \& Montoya, P. (2017). Sierra del Colmenar 1A, a new late Messinian (Late Miocene) locality in the Bajo Segura Basin (SE Spain): biostratigraphic and palaeoenvironmental implications. Historical Biology, 1-12. https://doi.org/10.1080/08 912963.2017.1297991

García-García, F.; Corbí, H.; Soria, J.M. \& Viseras, C. (2011). Architecture analysis of a river flood-dominated delta during an overall sea-level rise (Early Pliocene, SE Spain). Sedimentary Geology, 237: 102113. https://doi.org/10.1016/j.sedgeo.2011.02.010

Gennari, R.; Manzi, V.; Angeletti, L.; Bertini, A.; Biffi, U.; Ceregato, A.; Costanza, F.; Gliozzi, E.; Lugli, S.; Menichetti, E.; Rosso, A.; Roveri, M. \& Taviani, M. (2013). A shallow water record of the onset of the Messinian salinity crisis in the Adriatic foredeep (Legnagnone section, Northern Apennines). Palaeogeography, Palaeoclimatology, Palaeoecology, 386: 145-164. https://doi.org/10.1016/j.palaeo.2013.05.015

Gliozzi, E. \& Grossi, F. (2008). Late Messinian lagomare ostracod palaeoecology: A correspondence analysis approach. Palaeogeography, Palaeoclimatology, Palaeoecology, 264(3): 288-295. https://doi. org/10.1016/j.palaeo.2007.03.055

Hammer, Ø. \& Harper, D.A.T. (2006). Paleontological Data Analysis. Blackwell, 351 pp.

Hammer, Ǿ.; Harper, D.A.T. \& Ryan, P.D. (2001). PAST Palaeontological Statistics. Software package for education and data analysis. Paleontologia electronica, 4 (1): $1-9$

Herkat, M. (2007). Application of correspondence analysis to palaeobathymetric reconstruction of Cenomanian and Turonian (Cretaceous) rocks of Eastern Algeria. Palaeogeography, Palaeoclimatology, Palaeoecology, 254(3): 583-605. https://doi.org/10.1016/j. palaeo.2007.07.011

Herkat, M. \& Ladjal, A. (2013). Paleobathymetry of foraminiferal assemblages from the Pliocene of the Western Sahel (North-Algeria). Palaeogeography, Palaeoclimatology, Palaeoecology, 374: 144-163. https://doi.org/10.1016/j.palaeo.2013.01.013

Hinsbergen van, D.J.J.; Kouwenhoven, T.J. \& van der Zwaan, G.J. (2005). Paleobathymetry in the backstripping procedure: Correction for oxygenation effects on depth estimates Palaeogeography, Palaeoclimatology, Palaeoecology, 221: 245-265.https:// doi.org/10.1016/j.palaeo.2005.02.013

Jongman, R.H.G.; Braak C.J.F. ter \& van Fongeren, O.F.R. (1995). Data analysis in community and landscape ecology. Cambridge University Press, Cambrige, 299 pp.

Jorissen, F.J.; deStigter, H.C. \& Widmark, J.G.V. (1995). A conceptual model explaining benthic foraminiferal microhabitats: Marine Micropaleontology, 26: 3-15. https://doi.org/10.1016/0377-8398(95)00047-X

Martínez-Martínez, J.; Corbí, H.; Martin-Rojas, I.; BaezaCarratalá, J. F. \& Giannetti, A. (2017). Stratigraphy, petrophysical characterization and 3D geological modelling of the historical quarry of Nueva Tabarca island (western Mediterranean): Implications on heritage conservation. Engineering Geology, In press. https://doi.org/10.1016/j.enggeo.2017.10.014

Martin, R.E. (Ed.). (2000). Environmental micropaleontology: the application of microfossils to environmental geology. Topics in Geobiology, 15. New York, NY: Springer Science \& Business Media, 481 pp. https://doi.org/10.1007/978-1-4615-4167-7

Murray, J.W. (1991). Ecology and paleoecology of benthic foraminifera. N.Y. Longman Scientific and Technical, New York, 397 pp.

Murray, J.W. (2006). Ecology and applications of benthic foraminifera. Cambridge University Press, Cambrige, 426 pp. https://doi.org/10.1017/CBO9780511535529

Shi,G.R.(1993):Multivariatedataanalysisinpalaeoecology and palaeobiogeography - a review. Palaeogeography, Palaeoclimatology, Palaeoecology ,105: 199-234. https://doi.org/10.1016/0031-0182(93)90084-V

Soria, J.M.; Giannetti, A.; Monaco, P.; Corbí, H.; García-Ramos, D. \& Viseras, C. (2014). Cyclically-arranged, storm-controlled, prograding lithosomes in Messinian terrigenous shelves (Bajo Segura Basin, western Mediterranean). Sedimentary Geology, 310: 1-15. https://doi.org/10.1016/j.sedgeo.2014.06.001

Soria, J.M.; Caracuel, J.E.; Corbí, H.; Dinarès-Turell, J.; Lancis, C.; Tent-Manclús, J.E.; Viseras, C. \& Yébenes, A. (2008 a). The Messinian-Early Pliocene stratigraphic record in the southern Bajo Segura Basin (Betic Cordillera, Spain). Implications for the Mediterranean salinity crisis. Sedimentary Geology, 203: 267-288. https://doi.org/10.1016/j. sedgeo.2007.12.006

Soria, J.M.; Caracuel, J.E.; Corbí,H.; Dinarès-Turell, J.; Lancis, C.; Tent-Manclús, J.E. \& Yébenes, A. (2008 b). The Bajo Segura Basin (SE Spain): implications for the Messinian Salinity Crisis in the Mediterranean margins. Stratigraphy, 5: 259-265.

van der Zwaan, G.J.; Duijnstee, I.A.P.; den Dulk, M.; Ernst, S.R.; Jannik, N.T. \& Kouwenhoven, T.J. (1999). Benthic foraminifers: proxies or problems? A review of paleocological concepts. Earth Science 
Reviews, 46: 213-236. https://doi.org/10.1016/ S0012-8252(99)00011-2

Viseras, C.; Soria, J.M. \& Fernández, J. (2004): Cuencas Neógenas Postorogénicas de la Cordillera Bética. 576-581. In: Geología de España, Vera, J.A. (Ed), 890 pp.
Walid A. Makled, Medhat M.M. Mandur, \& Langer, M.R. (2017). Neogene sequence stratigraphic architecture of the Nile Delta, Egypt: A micropaleontological perspective. Marine and Petroleum Geology, 85: 117-135. https://doi.org/10.1016/j. marpetgeo.2017.04.017 